\title{
Safety and efficacy assessment of carotid artery stenting in a high-risk population in a single-centre registry
}

\author{
Michał Kosowski ${ }^{1,2}$, Wojciech Zimoch ${ }^{1,2}$, Tomasz Gwizdek ${ }^{3}$, Radosław Konieczny ${ }^{3}$, Piotr Kübler ${ }^{1,2}$, \\ Artur Telichowski ${ }^{2}$ Ewa A. Jankowska ${ }^{1,2}$, Krzysztof Reczuch ${ }^{1,2}$ \\ ${ }^{1}$ Department of Heart Diseases, Wroclaw Medical University, Wroclaw, Poland \\ ${ }^{2}$ Centre for Heart Diseases, $4^{\text {th }}$ Military Hospital, Wroclaw, Poland \\ ${ }^{3}$ Students' Scientific Group of Interventional Cardiology, Department of Heart Diseases, Wroclaw Medical University, Wroclaw, Poland
}

Postep Kardiol Inter 2014; 10, 4 (38): 258-263

DOI: $10.5114 /$ pwki.2014.46767

\begin{abstract}
A b stract
Introduction: Ischaemic stroke is the primary cause of long-term disability and the third most common cause of death. Internal carotid artery stenosis is an important risk factor for stroke and transient ischaemic attack (TIA). European Society of Cardiology (ESC) and American Heart Association (AHA) guidelines allow carotid artery stenting (CAS) as an alternative to endarterectomy in centres with low rates of death or stroke.

Aim: To assess the safety and efficacy of CAS in a single-centre observation.

Material and methods: We performed a retrospective analysis of all patients treated with CAS between March 2008 and July 2012. Clinical data and outcomes in both asymptomatic and symptomatic patients were analysed.

Results: A total of 214 consecutive patients were included in the registry. Symptomatic patients accounted for $57 \%$ of the study group and were more likely to have a history of stroke and/or TIA that occurred more than 6 months before the procedure (50\% vs. $8 \%, p<0.001)$. Asymptomatic patients were more likely to have a history of coronary artery disease (88\% vs. $61 \%, p<0.001)$, and the rates of previous acute coronary syndrome and revascularisation were also higher in this group (58\% vs. $41 \%$ and $71 \%$ vs. $52 \%$, respectively, both $p<0.05)$. The symptomatic group had higher incidence of stroke in periprocedural and $30-$ day observation $(4 \%$ vs. $0 \%, p<0.05)$. There was no difference in incidence of adverse events in long-term observation.

Conclusions: Carotid artery stenting is a safe and efficacious procedure. Every centre performing CAS should monitor the rate of periprocedural complications.
\end{abstract}

Key words: carotid artery stenting, peripheral artery disease.

\section{Introduction}

Cardiovascular diseases are the leading cause of mortality and disability in Europe. Ischaemic stroke is the most common cause of long-term disability and the third most common cause of death [1]. In various analyses, stroke mortality ranges from $10 \%$ to $30 \%$. One of the most important risk factors for stroke and transient ischaemic attack (TIA) is internal carotid artery stenosis [2]. In the majority of cases, carotid artery stenosis is caused by atherosclerosis. Some trials, such as NASCET and ACAS, showed benefits of carotid endarterectomy (CEA) over medical therapy [3]. Carotid artery stenting (CAS) is a less invasive method of carotid revascularisation, devoid of some complications that are typical for carotid endarterectomy (e.g. peripheral nerve damage), with a relatively high restenosis rate, and with comparable outcomes [4-6]. European Society of Cardiology (ESC) and American Heart Association (AHA) guidelines state that CAS may be considered as an alternative to CEA in high-volume centres with documented death or stroke rate $<6 \%$ in symptomatic patients and $<3 \%$ (or "low" according to the AHA guidelines) in asymptomatic patients $[7,8]$.

\section{Aim \\ The aim of this registry was to assess the safety and efficacy of CAS procedures performed in our centre in periprocedural, 30-day, and long-term periods.}




\section{Material and methods}

\section{Study design}

This was a single-centre, retrospective, registry-based study. The study protocol was approved by the local ethics committee and was in accordance with the Declaration of Helsinki.

\section{Registry group}

In our study we included all consecutive patients who underwent CAS procedures in our centre from March 2008 to July 2012.

In order to assess the significance of artery stenosis every patient had a duplex ultrasound performed. Asymptomatic patients with $70-99 \%$ and symptomatic patients with $50-99 \%$ carotid artery stenosis were selected for the procedure. According to the European Society of Cardiology guidelines on peripheral artery disease, carotid artery stenosis is considered symptomatic in the presence of TIA or stroke (affecting the corresponding central nervous system territory) within the previous 6 months [7].

All the comorbidities (heart failure, coronary artery disease, hypertension, dyslipidaemia, diabetes mellitus, chronic kidney disease, and peripheral artery disease) were diagnosed according to current guidelines.

\section{Procedure}

Every patient was examined by a neurologist before and after the procedure. Each patient was given standard medical pretreatment with antiplatelet agents (aspirin and clopidogrel). Anti-hypertensive drugs were withdrawn $24 \mathrm{~h}$ prior to the procedure. Procedure efficacy was defined as a successful stent deployment. All of the patients were discharged home on dual anti-platelet therapy and statins, unless there were contraindications. Every CAS procedure was performed by experienced operators.

Procedures were performed via femoral access. Proximal (Mo.Ma, Medtronic, USA) or distal (FilterWire EZ, Boston Scientific, USA) protection devices were used in every procedure, unless the operator was unable to introduce the device. Each patient was given 5000 IU of unfractionated heparin intravenously with an additional dose dependent on activated clotting time (ACT). Protege RX (ev3 Endovascular, USA), Cristallo Ideale (Medtronic, USA), Wallstent (Boston Scientific, USA), and RX Acculink (Abbott Vascular, USA) stents were used. Pre- and post-dilation were performed if needed. In cases of hypotonia the patient was given intravenous infusion of saline with dopamine when needed; bradycardia was treated with intravenous atropine injection. After the procedure every patient was monitored for $24 \mathrm{~h}$ in an Intensive Cardiac Care Unit.

\section{Adverse events}

Ischaemic stroke was defined as an acute neurological ischaemic event of at least $24 \mathrm{~h}$ duration with focal signs and symptoms, and TIA as temporary focal brain or retinal deficits caused by vascular disease that clear completely in less than $24 \mathrm{~h}$. Diagnosis of myocardial infarction was based on clinical history of chest pain, electrocardiographic changes, and serum cardiac enzymes. Contrast-induced nephropathy (CIN) was defined as either a greater than $25 \%$ increase of serum creatinine or an absolute increase in serum creatinine of $0.5 \mathrm{mg} / \mathrm{dl}$.

\section{Statistical analysis}

Quantitative values with normal distribution are shown as mean \pm standard deviation and were compared using Student's $t$-test. The $\chi^{2}$ test was used to analyse categorical data. For all tests a value of $p<0.05$ was considered statistically significant. Statistica 10.0 (StatSoft, USA) package was used to perform all statistical analyses. Separate analyses were done for the symptomatic and asymptomatic patient sub-groups.

\section{Results}

\section{Whole studied population}

A total of 214 consecutive patients, aged $68 \pm 9$ years, were included in the registry. Women accounted for $33 \%$ of the registry population. One hundred twenty-one patients (57\%) were symptomatic, according to ESC guidelines criteria. Older patients ( $\geq 75$ years) accounted for $25 \%$ of the registry population, $36 \%$ of patients had diabetes, $46 \%$ dyslipidaemia, $73 \%$ a history of coronary artery disease (CAD), $48 \%$ were survivors of acute coronary syndrome (ACS), $27 \%$ had been diagnosed with heart failure, $20 \%$ had peripheral artery disease, 32\% had survived a TIA or stroke more than 6 months before the procedure, $82 \%$ had arterial hypertension, $16 \%$ had chronic kidney disease (CKD), $60 \%$ of the registry population underwent coronary revascularisation procedures, and $14 \%$ were active smokers (Table I).

Mean internal carotid artery stenosis was $88 \pm 9 \%$. Left internal carotid artery (LICA) was the target vessel in $53 \%$ of procedures, and right internal carotid artery (RICA) in $47 \%$ of procedures. Contralateral total occlusion was observed in $4 \%$ of cases.

We observed a $96 \%$ procedure efficacy. In the vast majority of cases (98\%) only one stent was implanted, and 5 patients (2\%) required two stents. In $61 \%$ of procedures a predilation was performed. Post-dilation was done in $90 \%$ of procedures. We used embolic protection devices in $98 \%$ of procedures (distal $-86 \%$, proximal $14 \%)$. In 4 cases (2\%) the operator was unable to place the embolic protection device in the desired position, so the procedure was terminated.

Median of follow-up was 463 days. Six patients (2\%) were lost to follow-up. In periprocedural and 30-day period, stroke occurred in 5 patients (2\%), TIA in 10 patients (5\%), and acute myocardial infarction in 1 patient (0.5\%). There was no case of death during periprocedural and 
Table I. Baseline clinical data of the study group

\begin{tabular}{|c|c|c|c|}
\hline Parameter & $\begin{array}{l}\text { All patients } \\
(n=214)\end{array}$ & $\begin{array}{c}\text { Symptomatic patients } \\
(n=121)(57 \%)\end{array}$ & $\begin{array}{c}\text { Asymptomatic patients } \\
(n=93)(43 \%)\end{array}$ \\
\hline Age, mean \pm SD [years] & $68 \pm 9$ & $67 \pm 9$ & $69 \pm 9$ \\
\hline Age - older (> 75 years), $n(\%)$ & $53(25)$ & $22(18)$ & $31(33)$ \\
\hline Sex - female, $n(\%)$ & $70(33)$ & $38(31)$ & $32(34)$ \\
\hline $\mathrm{DM}, n(\%)$ & $77(36)$ & $37(31)$ & $40(43)$ \\
\hline Dyslipidaemia, $n(\%)$ & $99(46)$ & $55(45)$ & $44(47)$ \\
\hline CAD, $n(\%)$ & $156(73)$ & $74(61)$ & $82(88)^{\star \star \star}$ \\
\hline Previous ACS, $n$ (\%) & $103(48)$ & $49(41)$ & $54(58)^{*}$ \\
\hline Previous PCI or CABG, $n(\%)$ & $129(60)$ & $63(52)$ & $66(71)^{*}$ \\
\hline Heart failure, $n(\%)$ & $58(27)$ & $29(24)$ & $29(31)$ \\
\hline Peripheral artery disease, $n(\%)$ & $42(20)$ & $27(22)$ & $15(16)$ \\
\hline Previous (> 6 months) stroke and/or TIA, $n(\%)$ & $68(32)$ & $61(50)^{\star \star \star}$ & $7(8)$ \\
\hline Hypertension, $n(\%)$ & $175(82)$ & $102(84)$ & $73(78)$ \\
\hline CKD, $n(\%)$ & $33(16)$ & $20(17)$ & $14(15)$ \\
\hline Active smokers, $n$ (\%) & $30(14)$ & 13 (11) & 17 (18) \\
\hline
\end{tabular}

Data are presented as numbers and percentages for categorical variables. Quantitative values with normal distribution are shown as mean \pm standard deviation. ${ }^{*} p<0.05,{ }^{* * *} p<0.001$. DM - diabetes mellitus, CAD - coronary artery disease, ACS - acute coronary syndrome, TIA - transient ischaemic attack, CKD - chronic kidney disease, $\mathrm{PCl}$ - percutaneous coronary intervention, $C A B G$ - coronary artery bypass graft

30-day period; we did not record any case of intracranial haemorrhage either.

In the periprocedural period we observed 7 cases (3\%) of CIN, 1 patient $(0.5 \%)$ required pacemaker implantation due to persistent symptomatic bradycardia, and 3 patients (1\%) required dopamine infusion due to hypotonia. There were 2 patients (1\%) with haematoma located in the arterial access site, requiring either thrombin injection or surgical treatment (Table II).

In long-term follow-up 8 patients (4\%) died (3 patients died from cardiovascular events, 5 patients died from non-cardiovascular related causes 10 patients (5\%) suffered a stroke, 11 (5\%) had a TIA, and 6 patients (3\%) had an acute myocardial infarction (Table III).

\section{Symptomatic vs. asymptomatic patients}

Symptomatic patients were more likely to have a history of stroke and/or TIA that occurred more than 6 months before the procedure (50\% vs. $8 \%, p<0.001)$. Asymptomatic patients were more likely to have had CAD diagnosed previously ( $88 \%$ vs. $61 \%, p<0.001)$. The rates of previous ACS and coronary revascularisation (coronary artery bypass graft - CABG or percutaneous coronary intervention $-\mathrm{PCl}$ ) were also higher in this group (58\% vs. $41 \%$ and $71 \%$ vs. $52 \%$, respectively, both $p<0.05)$. These findings can be related to the fact that patients treated due to ACS or stable angina are also screened for atherosclerosis in other vascular areas and asymptomatic carotid artery stenosis can be found; furthermore, some symptomatic patients are referred for CAS from neurology departments, and their history of cardiovascular diseases is often negative.

We recorded no difference in procedural efficacy or periprocedural complications. The symptomatic group had higher incidence of stroke in periprocedural and 30-day observation ( $4 \%$ vs. $0 \%, p<0.05$ ). There was no difference in incidence of adverse events in long-term follow-up.

\section{Discussion}

Both European and American guidelines recommend CAS as a valuable alternative to CEA, with remarks that such procedures should be done by a team of well-qualified and experienced operators in high-volume centres with documented low rates of death or stroke [7, 8]. European guidelines require periprocedural death or stroke rates to be less than $6 \%$ in symptomatic patients (class of recommendation: $\mathrm{llb}$ ) and $3 \%$ in asymptomatic patients (class of recommendation: IIb). American guidelines are more favourable towards CAS with Class I recommendation for symptomatic patients (with low rate of death or stroke) and with Class Ila recommendation for asymptomatic ones. These recommendations were based on large trials, like CAVATAS, EVA-3S, ICSS, SPACE, SAPPHIRE, and CREST [6, 9-19] and meta-analyses of randomised trials. One of the meta-analyses, done by Economopoulos et al., involving 7484 patients, sum- 
Table II. Periprocedural and 30-day results

\begin{tabular}{|c|c|c|c|}
\hline Parameter & $\begin{array}{l}\text { All patients } \\
(n=214)\end{array}$ & $\begin{array}{l}\text { Symptomatic patients } \\
\quad(n=121)(57 \%)\end{array}$ & $\begin{array}{l}\text { Asymptomatic patients } \\
\quad(n=93)(43 \%)\end{array}$ \\
\hline Internal carotid artery stenosis, mean \pm SD (\%) & $88 \pm 9$ & $88 \pm 10$ & $88 \pm 7$ \\
\hline Contralateral total occlusion, $n(\%)$ & $8(4)$ & $7(6)$ & $1(1)$ \\
\hline Efficacy, $n(\%)$ & $206(96)$ & $117(97)$ & $89(96)$ \\
\hline Any death, $n(\%)$ & 0 & 0 & 0 \\
\hline Cardiovascular death, $n$ (\%) & 0 & 0 & 0 \\
\hline Stroke, $n(\%)$ & $5(2)$ & $5(4)^{*}$ & 0 \\
\hline $\mathrm{TIA}, n(\%)$ & $10(5)$ & $7(6)$ & $3(3)$ \\
\hline Intracranial haemorrhage, $n$ (\%) & 0 & 0 & 0 \\
\hline MI, $n(\%)$ & $1(0.5)$ & $1(1)$ & 0 \\
\hline $\mathrm{CIN}, n(\%)$ & $7(3)$ & $4(3)$ & $3(3)$ \\
\hline Bradycardia requiring PM implantation, $n(\%)$ & $1(0.5)$ & $1(1)$ & 0 \\
\hline Hypotonia requiring dopamine infusion, $n(\%)$ & $3(1)$ & $2(2)$ & 1 (1) \\
\hline $\begin{array}{l}\text { Access site hematoma requiring intervention, } \\
n(\%)\end{array}$ & $2(1)$ & 0 & $2(2)$ \\
\hline
\end{tabular}

Data are presented as numbers and percentages for categorical variables. Quantitative values with normal distribution are shown as mean \pm standard deviation. ${ }^{*} p<0.05$, TIA - transient ischaemic attack, MI-myocardial infarction, CIN - contrast induced nephropathy, PM - pacemaker

Table III. Long-term results

\begin{tabular}{lccc} 
Parameter & $\begin{array}{c}\text { All patients } \\
(n=214)\end{array}$ & $\begin{array}{c}\text { Symptomatic patients } \\
(n=121)(57 \%)\end{array}$ & $\begin{array}{c}\text { Asymptomatic patients } \\
(n=93)(43 \%)\end{array}$ \\
\hline Any death, $n(\%)$ & $8(4)$ & $4(3)$ & $4(4)$ \\
\hline Cardiovascular death, $n(\%)$ & $3(1)$ & $3(2)$ & 0 \\
\hline Stroke, $n(\%)$ & $10(5)$ & $6(5)$ & $4(4)$ \\
\hline TIA, $n(\%)$ & $11(5)$ & $7(6)$ & $4(5)$ \\
\hline Intracranial haemorrhage, $n(\%)$ & 0 & 0 & 0 \\
\hline MI, $n(\%)$ & $6(3)$ & $3(2)$ & $3(3)$
\end{tabular}

Data are presented as numbers and percentages for categorical variables. Quantitative values with normal distribution are shown as mean \pm standard deviation. TIA - transient ischaemic attack, MI - myocardial infarction

marised that outcomes of CAS compared to CEA were associated with increased risk of any stroke, decreased risk of periprocedural myocardial infarction, and statistically non-significant increase in total mortality [20].

The results of our registry-based analysis show that it is possible to maintain a low rate of periprocedural adverse events and achieve a high efficacy rate due to highly-experienced operators and appropriate patient selection.

In our registry population $57 \%$ of patients were symptomatic according to ESC guidelines criteria. Among the trials mentioned above, only the SAPPHIRE and CREST studies enrolled both symptomatic and asymptomatic patients. The SAPPHIRE and CREST populations were quite similar to the one observed in our registry, although there were differences regarding age (older SAPPHIRE population), prevalence of dyslipidaemia (more frequent in CREST and SAPPHIRE populations), diabetes (less frequent in SAPPHIRE population), chronic kidney disease (less frequent in SAPPHIRE population), and coronary artery disease with CABG/PCI (more frequent in SAPPHIRE patients). The meta-analysis by Economopoulos et al. analysed data from 13 trials and found that $80 \%$ of patients were symptomatic. There was no direct comparison between results in asymptomatic and symptomatic sub-groups. In our registry population, we found higher incidence of stroke in symptomatic patients during the periprocedural period, but the groups did not differ in long-term follow-up.

Some meta-analyses and registries have shown that there is no a great impact of age on complications rate 
after CEA [21]; however, older patients were always considered at high surgical risk. The latter opinion was shared by some authors, and their conclusion therefore was that older patients could be good candidates for CAS procedure [22-24]. In our material older patients (> 75 years old) accounted for $25 \%$ of the population, with no difference among symptomatic and asymptomatic patients. Contrary to the opinions mentioned above, some authors emphasise that in older patients the procedure can be more challenging and related to higher rates of periprocedural complications caused by severely calcified vessels and vessel tortuosity $[25,26]$. To minimise the risk of distal embolisation, guidelines suggest that use of embolic protection devices (EPD) may be considered (class of recommendation: $\mathrm{llb}$ ).

A single-centre, randomised study with a small number of patients (36) carried out by Barbato et al. showed no reduction of microembolisation during CAS with EPD [27]. In the trial by Macdonald et al. CAS with EPD was associated with an increase in new lesions on diffusion-weighted magnetic resonance imaging, and significantly higher rates of microembolisation on transcranial Doppler [28]. In the SPACE and ICSS trials EPD use was not mandatory, and both studies showed no benefit from EPD use [4, 29]. Although the outcomes with use of EPD in the publications mentioned above were controversial, the best results of CAS were observed in trials and registries with mandatory use of EPD (SAPPHIRE, CREST, two large registries by Stabile et al. and Zahn et al.) [5, 6, 30, 31]. In our registry EPD use was mandatory; in 4 cases when it was impossible to introduce the device, and the procedure was not continued.

Women accounted for $33 \%$ of our registry population, which is similar to data from other trials and registries. An increased risk of CEA perioperative complications among women was reported $[32,33]$. The impact of sex on outcomes of CAS remains uncertain. In a systematic review of trials by Touzé et al. there was no evidence that there was increased risk in women. In our registry group women were less likely to have a history of hypertension and chronic kidney disease, but these differences did not affect outcome - there was no difference in adverse event rates between men and women [34].

Other factors, commonly found in our registry patients, such as a history of CAD (73\%), acute coronary syndrome (48\%), heart failure (27\%), previous stroke and/ or TIA (32\%), arterial hypertension ( $82 \%)$, chronic kidney disease $(16 \%)$, and previous $\mathrm{PCI}$ or CABG $(60 \%)$, are widely acknowledged risk factors for any operation, for those high-risk patients the guidelines recommend CAS as an alternative to CEA (class of recommendation: Ila).

In 2007 Ochała et al. published the results of a registry-based observation of Polish high-risk patients qualified to CABG surgery, who underwent CAS procedure with mandatory use of EPD due to significant internal carotid artery stenosis. Even though those patients were a selected group of high-risk patients awaiting CABG sur- gery due to advanced coronary artery disease, the general characteristics of the registry group were similar to our population. The incidence of adverse cardiovascular events, partially connected with elective CABG surgery, was similar to the results of trials and registries involving high-risk patients, as well as to our registry [35].

We could not perform appropriate statistical analyses for each of the sub-groups mentioned above due to the limited number of patients and relatively low rate of adverse events.

\section{Conclusions}

Carotid artery stenting, even in a high-risk population, is a relatively safe and efficacious procedure. Every centre performing CAS procedures should constantly monitor the rate of periprocedural complications because outcomes of CAS - like every relatively new method depend mainly on the experience of the operators and appropriate patient selection.

\section{References}

1. Lloyd-Jones D, Adams RJ, Brown TM, et al. American Heart Association Statistics Committee and Stroke Statistics Subcommittee. Heart disease and stroke statistics: 2010 update: a report from the American Heart Association. Circulation 2010; 121 : e46-215.

2. Grau AJ, Weimar C, Buggle F, et al. Risk factors, outcome, and treatment in subtypes of ischemic stroke: the German stroke data bank. Stroke 2001; 32: 2559-66.

3. Barnett HJ, Taylor DW, Eliasziw M, et al. Benefit of carotid endarterectomy in patients with symptomatic moderate or severe stenosis. North American Symptomatic Carotid Endarterectomy Trial Collaborators. N Engl J Med 1998; 339: 1415-25.

4. Bonati LH, Jongen LM, Haller S, et al. New ischaemic brain lesions on MRI after stenting or endarterectomy for symptomatic carotid stenosis: a substudy of the International Carotid Stenting Study (ICSS). Lancet Neurol 2010; 9: 353-62.

5. Massop D, Dave R, Metzger C, et al. Stenting and angioplasty with protection in patients at high-risk for endarterectomy: SAPPHIRE Worldwide Registry first 2,001 patients. Catheter Cardiovasc Interv 2009; 73: 129-36.

6. Brott TG, Hobson RW 2nd, Howard G, et al. Stenting versus endarterectomy for treatment of carotid-artery stenosis. N Engl J Med 2010; 363: 11-23.

7. European Stroke Organisation, Tendera M, Aboyans V, Bartelink ML, et al. ESC Committee for Practice Guidelines. ESC Guidelines on the diagnosis and treatment of peripheral artery diseases: document covering atherosclerotic disease of extracranial carotid and vertebral, mesenteric, renal, upper and lower extremity arteries: the Task Force on the Diagnosis and Treatment of Peripheral Artery Diseases of the European Society of Cardiology (ESC). Eur Heart J 2011; 32: 2851-906.

8. Brott TG, Halperin JL, Abbara S, et al. 2011 ASA/ACCF/AHA/ AANN/AANS/ACR/ASNR/CNS/SAIP/SCAI/SIR/SNIS/SVM/SVS guideline on the management of patients with extracranial carotid and vertebral artery disease: executive summary: a report of the American College of Cardiology Foundation/American Heart Association Task Force on Practice Guidelines, and the 
American Stroke Association, American Association of Neuroscience Nurses, American Association of Neurological Surgeons, American College of Radiology, American Society of Neuroradiology, Congress of Neurological Surgeons, Society of Atherosclerosis Imaging and Prevention, Society for Cardiovascular Angiography and Interventions, Society of Interventional Radiology, Society of Neurolnterventional Surgery, Society for Vascular Medicine, and Society for Vascular Surgery. Developed in collaboration with the American Academy of Neurology and Society of Cardiovascular Computed Tomography. Catheter Cardiovasc Interv 2013; 81: E76-123.

9. Endovascular versus surgical treatment in patients with carotid stenosis in the Carotid and Vertebral Artery Transluminal Angioplasty Study (CAVATAS): a randomised trial. Lancet 2001; 357: 1729-37.

10. Mas JL, Chatellier G, Beyssen B, et al. Endarterectomy versus stenting in patients with symptomatic severe carotid stenosis. N Engl J Med 2006; 355: 1660-71.

11. Ederle J, Dobson J, Featherstone RL, et al. Carotid artery stenting compared with endarterectomy in patients with symptomatic carotid stenosis (International Carotid Stenting Study): an interim analysis of a randomised controlled trial. Lancet 2010; 375: 985-97.

12. Fiehler J, Jansen O, Berger J, Eckstein HH, Ringleb PA, Stingele R. Differences in complication rates among the centres in the SPACE study. Neuroradiology 2008; 50: 1049-53.

13. Yadav JS, Wholey MH, Kuntz RE, et al. Protected carotid-artery stenting versus endarterectomy in high-risk patients. N Engl J Med 2004; 351: 1493-501.

14. Gurm HS, Yadav JS, Fayad P, et al. Long-term results of carotid stenting versus endarterectomy in high-risk patients. N Engl J Med 2008; 358: 1572-9.

15. Ederle J, Bonati LH, Dobson J, et al. Endovascular treatment with angioplasty or stenting versus endarterectomy in patients with carotid artery stenosis in the Carotid and Vertebral Artery Transluminal Angioplasty Study (CAVATAS): long-term follow-up of a randomised trial. Lancet Neurol 2009; 8: 898-907.

16. Ringleb PA, Allenberg J, Bruckmann $\mathrm{H}$, et al. 30 day results from the SPACE trial of stent-protected angioplasty versus carotid endarterectomy in symptomatic patients: a randomised non-inferiority trial. Lancet 2006; 368: 1239-47.

17. Eckstein HH, Ringleb P, Allenberg JR, et al. Results of the Stent-Protected Angioplasty versus Carotid Endarterectomy (SPACE) study to treat symptomatic stenoses at 2 years: a multinational, prospective, randomized trial. Lancet Neurol 2008; 7: 893-902.

18. Mas JL, Chatellier G, Beyssen B, et al. Carotid angioplasty and stenting with and without cerebral protection: clinical alert from the Endarterectomy Versus Angioplasty in Patients With Symptomatic Severe Carotid Stenosis (EVA-3S) trial. Stroke 2004; 35: e18-20.

19. Mas JL, Trinquart L, Leys D, et al. Endarterectomy Versus Angioplasty in patients with Symptomatic Severe Carotid Stenosis (EVA-3S) trial: results up to 4 years from a randomised, multicentre trial. Lancet Neurol 2008; 7: 885-92.

20. Economopoulos KP, Sergentanis TN, Tsivgoulis G, et al. Carotid artery stenting versus carotid endarterectomy: a comprehensive meta-analysis of short-term and long-term outcomes. Stroke 2011; 42: 687-92.
21. Bond R, Rerkasem K, Cuffe R, et al. A systematic review of the associations between age and sex and the operative risks of carotid endarterectomy. Cerebrovasc Dis 2005; 20: 69-77.

22. Safian RD, Bresnahan JF, Jaff MR, et al. CREATE Pivotal Trial Investigators. Protected carotid stenting in high-risk patients with severe carotid artery stenosis. J Am Coll Cardiol 2006; 47: 2384-9.

23. Gray WA, Yadav IS, Verta P, et al. The CAPTURE registry: results of carotid stenting with embolic protection in the post approval setting. Catheter Cardiovasc Interv 2007; 69: 341-8.

24. Katzen BT, Criado FJ, Ramee S, et al. CASES-PMS Investigators. Carotid artery stenting with emboli protection surveillance study: thirty-day results of the CASES-PMS study. Catheter Cardiovasc Interv 2007; 70: 316-23.

25. Bazan HA, Pradhan S, Mojibian H, et al. Increased aortic arch calcification in patients older than 75 years: implications for carotid artery stenting in elderly patients. J Vasc Surg 2007; 46: 841-5.

26. Lam RC, Lin SC, DeRubertis B, et al. The impact of increasing age on anatomic factors affecting carotid angioplasty and stenting. J Vasc Surg 2007; 45: 875-80.

27. Barbato JE, Dillavou E, Horowitz MB, et al. A randomized trial of carotid artery stenting with and without cerebral protection. J Vasc Surg 2008; 47: 760-5.

28. Macdonald S, Evans DH, Griffiths PD, et al. Filter-protected versus unprotected carotid artery stenting: a randomized trial. Cerebrovasc Dis 2010; 29: 282-9.

29. Jansen O, Fiehler J, Hartmann M, et al. Protection or non-protection in carotid stent angioplasty: the influence of interventional techniques on outcome data from the SPACE Trial. Stroke 2009; 40: 841-6.

30. Stabile E, Salemme L, Sorropago G, et al. Proximal endovascular occlusion for carotid artery stenting: results from a prospective registry of 1,300 patients. J Am Coll Cardiol 2010; 55: 1661-7.

31. Zahn R, Mark B, Niedermaier N, et al. Embolic protection devices for carotid artery stenting: better results than stenting without protection? Eur Heart I 2004; 25: 1550-8.

32. Rothwell PM, Eliasziw M, Gutnikov SA, et al. Carotid endarterectomy trialists collaboration. Endarterectomy for symptomatic carotid stenosis in relation to clinical subgroups and timing of surgery. Lancet 2004; 363: 915-24.

33. Brown MM, Raine R. Should sex influence the choice between carotid stenting and carotid endarterectomy? Lancet Neurol 2011; 10: 494-7.

34. Touzé E, Trinquart L, Felgueiras R, et al. Carotid Stenting Trialists' Collaboration. A clinical rule (sex, contralateral occlusion, age, and restenosis) to select patients for stenting versus carotid endarterectomy: systematic review of observational studies with validation in randomized trials. Stroke 2013; 44: 3394-400.

35. Ochała A, Kaźmierski M, Smolka G, et al. Carotid artery stenting with brain protection devices in cardiovascular high risk patients - 12 month follow-up. Post Kardiol Interw 2007; 3: 128-35. 